\title{
Infrared thermography for defect detection on aluminium-CFRP hybrid 3D structure
}

\author{
by H. C. Fernandes ${ }^{a, b}$, S. Quirin ${ }^{c}$, M. Schwarz c, b and H.-G. Herrmann b, c \\ a Federal University of Uberlandia, 38408-100, Uberlandia, Brazil. henrique.fernandes@ufu.br \\ ${ }^{b}$ Fraunhofer IZFP Institute for Nondestructive Testing, 66123 Saarbrucken, Germany \\ c Chair for Lightweight Systems, Saarland University, Campus E3 1, 66123 Saarbrucken, Germany
}

\begin{abstract}
Advanced materials are widely used in several industries. They play an important role especially in the aeronautic sector where weight reduction is required in order to reduce fuel consumption. Composite materials have a high strength to weight ratio and are applied in airplane construction. In order to ensure the safety of these components during their life cycle, non-destructive testing methods are required. In this work, infrared thermography is used to inspect an aluminiumCFRP hybrid 3D structure. A dual-band infrared camera is used to capture images in two different spectral ranges. Two image-processing techniques are then applied in order to enhance the images: principal component thermography and partial least squares thermography. Finally, a signal-to-noise ratio analysis is performed to assess the quality of the images and detected defects. Results showed that PCT has a slight advantage over PLST in our experiments.
\end{abstract}

\section{Introduction}

Carbon fibre reinforced polymer (CFRP) are widely used in aeronautical industries due to their excellent specific mechanical properties (i.e., property/density ratio) such as stiffness and strength, as well as relatively high temperature and oxidation resistances. However, to substitute all metal structures by CFRP structures is not viable. Hence, hybrid structures are usually used. In this work, an aluminium-CFRP hybrid 3D sample is studied. This hybrid is manufactured by injection moulding and the aluminium component in the CFRP creates an additional interface where defects are likely to occur. These defects may occur during fabrication or during their life cycle due to mechanical load. It is important to assess these defects and detect them in order to ensure the safety of the structures manufactured with such materials.

Infrared thermography (IRT) is a valuable non-destructive testing (NDT) tool used to assess this kind of damage. IRT is broadly used to inspect composite materials, especially CFRP. It is fast, non-contact and does not emit any harmful radiation. This topic has been explored extensively with several papers available in the literature [1-3].

In this work, IRT is applied to inspect an aluminium-CFRP hybrid 3D sample with internal defects. First, a Pulsed Thermography (PT) experiment in reflection mode is conducted. Then, a radiometric calibration is performed in the raw data which is acquired with the camera. To mitigate the effects of the 3D surface and the distortions of the camera, a geometric calibration of the camera is also performed. With the calibrated and corrected data, partial least squares thermography (PLST) and principal component thermography (PCT) are then applied to enhance the obtained PT signal for better defect visualization. The main advantage of both techniques is that they reduce the amount of data from thousand images to approximately 10 images. Finally, in order to quantify the visual quality of the defects detected, a signal-to-noise ratio analysis is conducted.

\section{Materials and methods}

\subsection{Inspected sample}

The sample inspected in this paper is a metal-CFRP-hybrid structure with a thermoplastic layer between CFRP and metal [3]. The metal inlay is a 3mm thick aluminium alloy (EN AW-6082) and is bended to reach a 3D geometry. The characteristic form is produced in a stamping process. In a further step, the metal insert is overmoulded with the thermoplastic component in an injection moulding process. The thermoplastic is a polyphthalamide (PPA) with 30 vol\% of glass fibre (Vestamid ${ }^{\circledR}$ HT plus M1033). In the last step, the overmoulded inlay is draped in the middle of four layers Cfibre canvas fabric. This composite is cured in a Resin Transfer Moulding (RTM) process for 20 minutes with a temperature of $75^{\circ} \mathrm{C}$ and a pressure of 8 bar to a thickness of $1 \mathrm{~mm}$. The used fibres are Torayca T300/ FT300, the CFRP-layers are arranged symmetrical as a $\left[0 / 90^{\circ}, \pm 45^{\circ}\right]_{s}$ laminate and it is used a $2 \mathrm{~K}$ hot curing epoxy system from SIKA (Biresin $\mathrm{CR} 170 / \mathrm{CH} 150-3)$. This hybrid structure reaches its strength due to a mechanical interlocking. The thermoplastic layer reduces the gap of stiffness between metal and CFRP and prevents corrosion as well. In this sample, interfaces, that are weak points in the structure, occur and must be characterised by non-destructive testing methods. Figure 1 shows an image of the sample. 


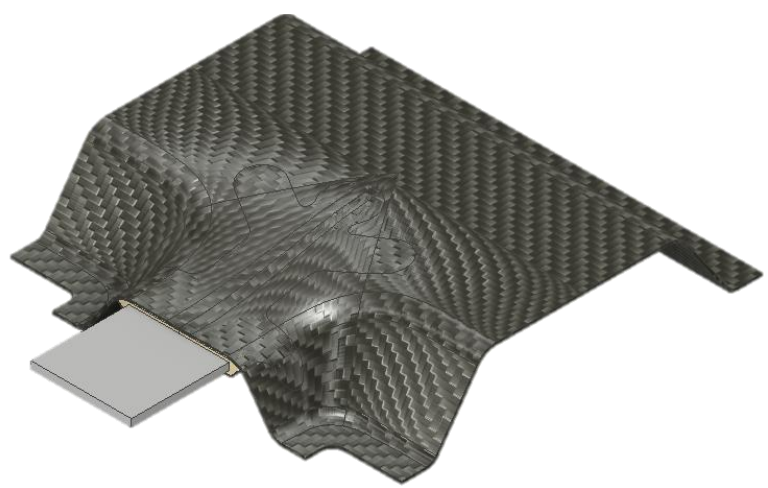

Figure 1. Inspected sample

\subsection{PT set-up}

In this work, inspections were performed using a PT setup in reflection mode (Figure 2). The setup had a 2009 Thermosensorik QWIP Dualband 384, an infrared dual-band camera working simultaneously in the $4.4-5.2 \mu \mathrm{m}$ (denoted as MWIR) and the $7.8-8.8 \mu \mathrm{m}$ (denoted as LWIR) bands. Each detector has an image frame size of $384 \times 288$ pixels. To heat up the sample, it is used a circular flash device with several lamps (3kJ). A short flash was fired for a period of $10 \mu s$ and the infrared camera recorded approximately $5 \mathrm{~s}$ of images at a frame rate of around $145 \mathrm{fps}$.



Figure 2. PT set-up used during the experiments.

\subsection{Radiometric calibration}

A radiometric calibration has been performed on the raw data from the camera. To get homogenous radiance at different temperatures, a black body radiation source is used. The advantage of using the raw data is, that you can include the calibration procedure wherever you like in your experiment and transfer it to all measurements taken before and after that. The effort to acquire the calibration points is further reduced by the method based on Planck's law proposed in [4], which only needs two calibration images whereas one was taken close to room temperature. The characteristic of a quantum detector is represented better by radiance than temperature values. Furthermore, the pixel-by-pixel calculation leads to sharper images because of its quality as a non-uniformity correction (NUC).

\subsection{Geometric correction}

Images captured by cameras usually contain distortions caused by the lenses of the camera. These distortions could introduce geometric anomalies on the captured images especially near the borders. For instance, a sharp line could 
appear curved in the image. Since a 3D sample is inspected, a geometric correction is applied. To perform this correction, it is mandatory to know the camera parameters (or intrinsic parameters). To determine these parameters a camera calibration has to be performed. The calibration process consists of identifying the internal quantities of the camera that affect the imaging process: image centre position (in pixels), focal length, different scaling factors for row pixels and column pixels, skew factor and lens distortion. In this context, camera calibration can be modelled as an optimisation process, where the discrepancy between the observed images features and their theoretical position is minimised with respect to the camera's intrinsic parameters. These calibration images are captured from a known object. In this project, different images of a chessboard are captured and the optimisation process is performed with these images. Details of the calibration process can be found in [5]. Figure 3 shows the difference between a corrected and an uncorrected image.

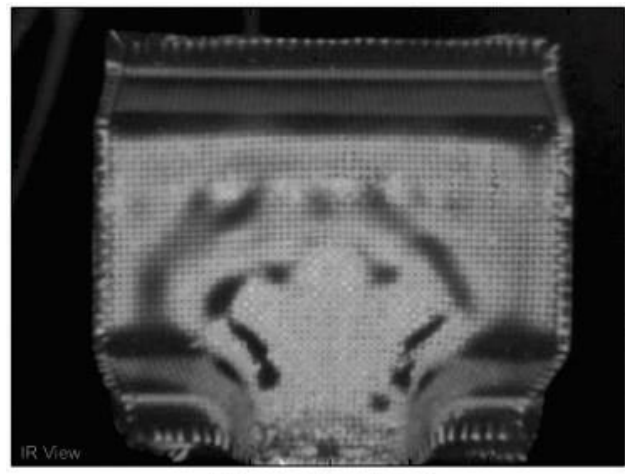

Uncorrected

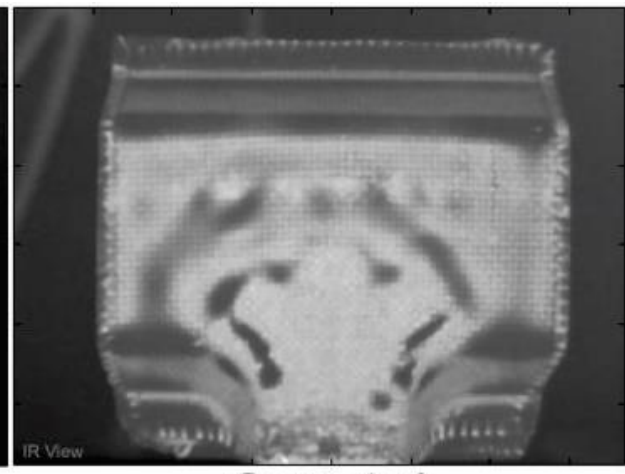

Corrected

Figure 3. IR images before and after geometric correction

\subsection{Principal component thermography (PCT)}

IR sequences often have a low signal-to-noise ratio and need to be enhanced for better visualization. In this work, a well-known processing technique is used: principal component thermography (PCT). PCT, originally proposed by Rajic in [6], extracts the image features and reduces the undesirable signals. It relies on Singular Value Decomposition (SVD), which is a tool to extract spatial and temporal data from a matrix in a compact manner by projecting original data onto a system of orthogonal components known as EOF. By sorting the principal components in such way that the first EOF represents the most characteristic variability of the data, the second EOF contains the second most important variability, and so on, the original data can be adequately represented with only a few EOFs.

\subsection{Partial least squares thermography (PLST)}

Based on a statistical correlation method, partial least squares thermography (PLST) has been proposed as a technique for the treatment of thermographic images [7]. PLST computes loading $P$ and score T vectors that are correlated to the predicted block $Y$ (as in maximum redundancy analysis), while describing a large amount of the variation in the predictor matrix $X$ (as in principal component regression). The matrix $X$ corresponds to the surface temperature matrix obtained during the PT inspection, while $Y$ is defined by the observation time during which the thermal images were captured. The PLS model is achieved by decomposing $X$ and $Y$ into a combination of loadings $P$ and $Q$ (formed by orthogonal vectors), scores $T$ and $U$ (the projections of the loading vectors associated with the singular values) and residuals $E$ and $F$. One of the main attractions of PLST is the separation of physical effects. This is because of the orthogonality between each latent variable obtained from the decomposition of the thermal sequence.

\subsection{Signal-to-noise ratio (SNR)}

A SNR value is used to determine which processing algorithm is more suitable for a certain application. To determine this SNR value, two areas in the resulting image obtained with the processing algorithm are selected: an area inside the defect and an area around it (sound area). In this work the following equation to compute SNR is used [8]:

$$
S N R=20 * \log _{10}\left(\frac{\text { abs }(\text { avg }(\text { area_defect })-\text { avg }(\text { area_sound }))}{\sigma}\right)[d B]
$$

where avg(area_defect) is the average value of the pixels of the defect, avg(area_sound) is the average value of the pixels of a sound area and $\sigma$ is the standard deviation of the sound area. 


\section{Results and discussion}

The sample is inspected with the PT setup shown in Figure 2. Since a dual-band IR camera is used, two different sets of images are acquired: LWIR sequence and MWIR sequence. Sequences are then radiometrically calibrated, and the geometric correction is applied in these calibrated data. Result image sequences are processed later with PCT and PSLT. Three defects are chosen to compare the quality of the images obtained with the two processing techniques. Figure 4 shows the obtained results.
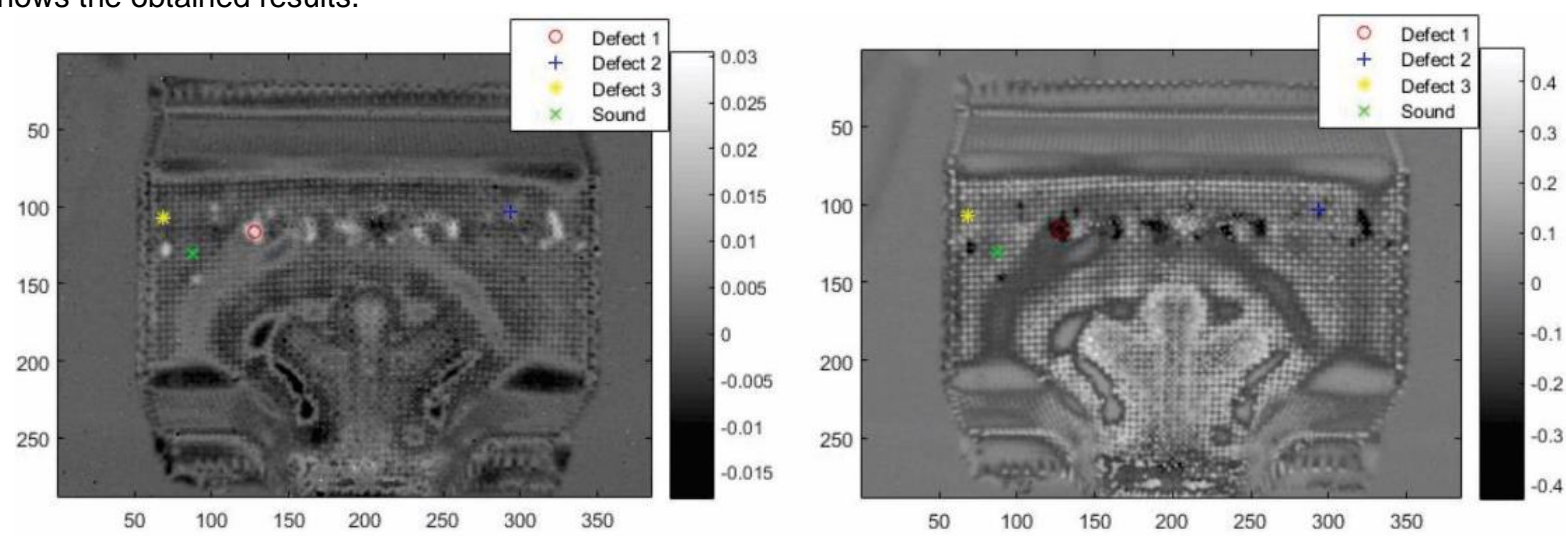

(a)
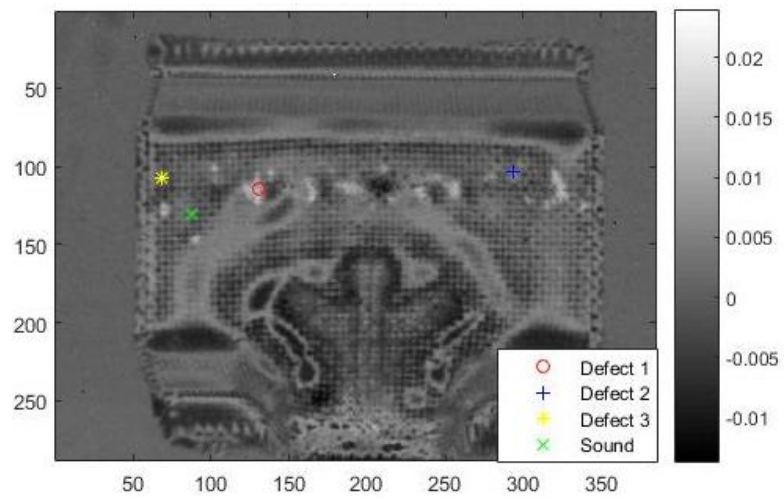

(b)

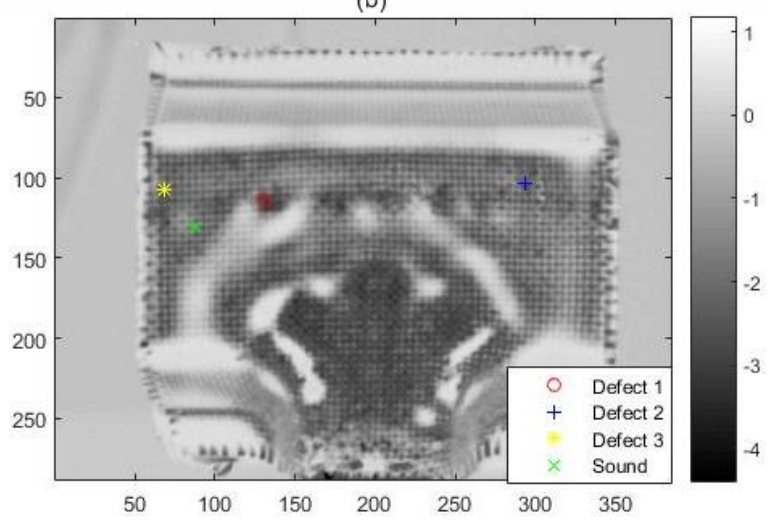

Figure 4. Result images. MWIR: (a) PCT third EOF, and (b) PLST: second loading. LWIR: (c) PCT third EOF, and (d) PLST: first loading

Results show that both PCT and PLST are able to detect several defects including the insertion on the upper part and problems with the aluminium and CFRP interface. Table 1 shows the SNR values of the three depicted defects for both techniques and the different sequences. Results show that, for our experiment, PCT provided a clearer image than PLST.

Table 1. SNR values

\begin{tabular}{|c|c|r|r|r|}
\cline { 2 - 5 } \multicolumn{1}{c|}{} & \multicolumn{2}{c|}{ MWIR } & \multicolumn{1}{c|}{ LWIR } \\
\hline Defect & PCT & PLST & PCT & PLST \\
\hline$\# 1$ & $19.7 \mathrm{~dB}$ & $17.1 \mathrm{~dB}$ & $17.6 \mathrm{~dB}$ & $13.2 \mathrm{~dB}$ \\
\hline$\# 2$ & $10.0 \mathrm{~dB}$ & $9.1 \mathrm{~dB}$ & $14.2 \mathrm{~dB}$ & $5.7 \mathrm{~dB}$ \\
\hline$\# 3$ & $11.7 \mathrm{~dB}$ & $9.8 \mathrm{~dB}$ & $14.8 \mathrm{~dB}$ & $12.3 \mathrm{~dB}$ \\
\hline
\end{tabular}

Additionally, mean and standard deviation values of the obtained SNRs could also be calculated. If only the SNR values obtained with PCT are considered, LWIR sequence has greater values. Mean and standard deviation for MWIR is 13.81 and $4.26 \mathrm{~dB}$ respectively, while for LWIR they are 15.55 and $1.49 \mathrm{~dB}$ respectively.

\section{Conclusions}

In this work, an aluminum-CFRP hybrid 3D sample with internal defects was inspected using pulsed thermography. Radiometric calibration and geometric correction were performed so that the data could be as closest as possible to what was happening in the sample. Treated data was then processed with two different IR processing techniques: PCT and PLST. Both techniques were able to detect the defects. Finally, a SNR analysis was performed which indicated that, for this experiment, LWIR sequences processed with PCT produced better results. Next steps of the 
research include adding a 3D geometric model of the sample for better defect visualization of the defects detected in the 2D infrared image.

\section{REFERENCES}

[1] H. Zhang, P. Verberne, S. A. Meguid, C. Ibarra-Castanedo, X. P. Maldague, Autonomous high resolution inspection of kiss-bonds skins of carbon nanotube reinforced nanocomposites using novel dynamic line-scan thermography approach, Composites Science and Technology 192:108111, 2020.

[2] X. Meng, Y. Wang, J. Liu, W. He, Nondestructive inspection of curved clad composites with subsurface defects by combination active thermography and three-dimensional (3d) structural optical imaging, Infrared Physics \& Technology 97:424 - 431, 2019.

[3] J. Summa, M. Becker, F. Grossmann, M. Pohl, M. Stommel, H.-G. Herrmann, Fracture analysis of a metal to cfrp hybrid with thermoplastic interlayers for interfacial stress relaxation using in situ thermography, Composite Structures 193:19-28, 2018.

[4] S. Quirin, H.-G. Herrmann, Combining the spectral information of dual-band images to enhance contrast and reveal details, in: Proceedings of the 14th International Conference on Quantitative Infrared Thermography 2018, June, Berlin, Germany (QIRT 2018), Creative Commons, pp. 779-785, 2018.

[5] H.-C. Fernandes, X. Maldague, Fiber orientation assessment in complex shaped parts reinforced with carbon fiber using infrared thermography, Quantitative InfraRed Thermography Journal 12: 64-79, 2015.

[6] N. Rajic, Principal component thermography for flaw contrast enhancement and flaw depth characterisation in composite structures. Composite Structures, 58(4):521-528, 2002.

[7] F. Lopez, Clemente Ibarra-Castanedo, Vicente de Paulo Nicolau, Xavier Maldague, Optimization of pulsed thermography inspection by partial least-squares regression. NDT \& E International, 66:128-138, 2014.

[8] P. Albendea, F. J. Madruga, A. Cobo, J. M. López-Higuera, Signal to noise ratio (SNR) comparison for pulsed thermographic data processing methods applied to welding defect detection, in: Proceedings of the $X$ International Conference on Quantitative InfraRed Thermography, pp. 27-30, 2010. 